\title{
Arsenic NQR in the Paramagnetic Complex $\mathrm{Co}\left(\mathrm{AsF}_{6}\right)_{2} \cdot 2 \mathrm{AsF}_{3}$
}

\author{
J. Pirnat ${ }^{\mathrm{a}}$, Z. Trontelj ${ }^{\mathrm{b}, \mathrm{a}}$, Z. Jagličića ${ }^{\mathrm{a}}, \mathrm{K}$. Lutar ${ }^{\mathrm{c}}$, and H. Borrmann ${ }^{\mathrm{d}}$ \\ ${ }^{a}$ Inst. of Mathematics, Physics and Mechanics, P. O. Box 2964, 1001 Ljubljana, Slovenia \\ ${ }^{\mathrm{b}}$ Faculty of Mathematics and Physics, Univ. of Ljubljana, Slovenia \\ ${ }^{c}$ Inst. Jožef Stefan, Ljubljana, Slovenia \\ ${ }^{\mathrm{d}}$ MPI für Chem. Physik fester Stoffe, Dresden, Germany \\ Reprint requests to Dr. J. P.; E-mail: janez.pirnat@imfm.uni-lj.si
}

Z. Naturforsch. 55 a, 212-214 (2000); received October 13, 1999

Presented at the XVth International Symposium on Nuclear Quadrupole Interactions, Leipzig, Germany, July 25 - 30, 1999.

Arsenic $\mathrm{NQR}$ in $\mathrm{Co}\left(\mathrm{AsF}_{6}\right)_{2} \cdot 2 \mathrm{AsF}_{3}$ reveals three lines: one near $122 \mathrm{MHz}$ and two near $50 \mathrm{MHz}$. Relations of the spectral lines to the structure and to thermal vibrations are discussed. Possible influence of paramagnetic Co is examined.

Key words: $\mathrm{Co}\left(\mathrm{AsF}_{6}\right)_{2}$; Complex; NQR; Paramagnet; Crystal Structure.

\section{Introduction}

Compounds of the type $\mathrm{M}\left(\mathrm{AsF}_{6}\right)_{2}(\mathrm{M}=\mathrm{Co}, \mathrm{Mn})$ are possible starting materials in fluorine chemistry for the preparation of new binary fluorides of transition metals in high oxidation states $[1,2]$. However, the use of these compounds requires their precise characterization. The application of As NQR spectroscopy is one possibility. This is the main purpose of our present research. On the other side, it is also a challenging task for an experimentalist to measure NQR in paramagnetic substances, which is usually hard to detect. If the NQR lines are not totally smeared out by the local magnetic fields of the paramagnetic ions in the crystal structure, they can serve as a probe for these magnetic fields.

A series of $\mathrm{M}\left(\mathrm{AsF}_{6}\right)_{2}$ complex compounds containing different ligands has been prepared at the Fluorochemistry Department of the Institute J. Stefan, Ljubljana, and for several of them the structures have been already solved. One of them is the complex $\mathrm{Co}\left(\mathrm{AsF}_{6}\right)_{2} \cdot 2 \mathrm{AsF}_{3}$ [2], where a broad frequency scan using a superregenerative $\mathrm{NQR}$ spectrometer revealed clear signals. Its structure is shown in Figure 1 . The structure is layered, each layer containing a two-dimensional square lattice, made of two kinds of slightly different but fairly regular octahedra $\mathrm{AsF}_{6}$ and $\mathrm{Co}$ atoms binding them at the nodes ( $\mathrm{Co}$ and As sites are exactly in the same plane). Two $\mathrm{AsF}_{3}$

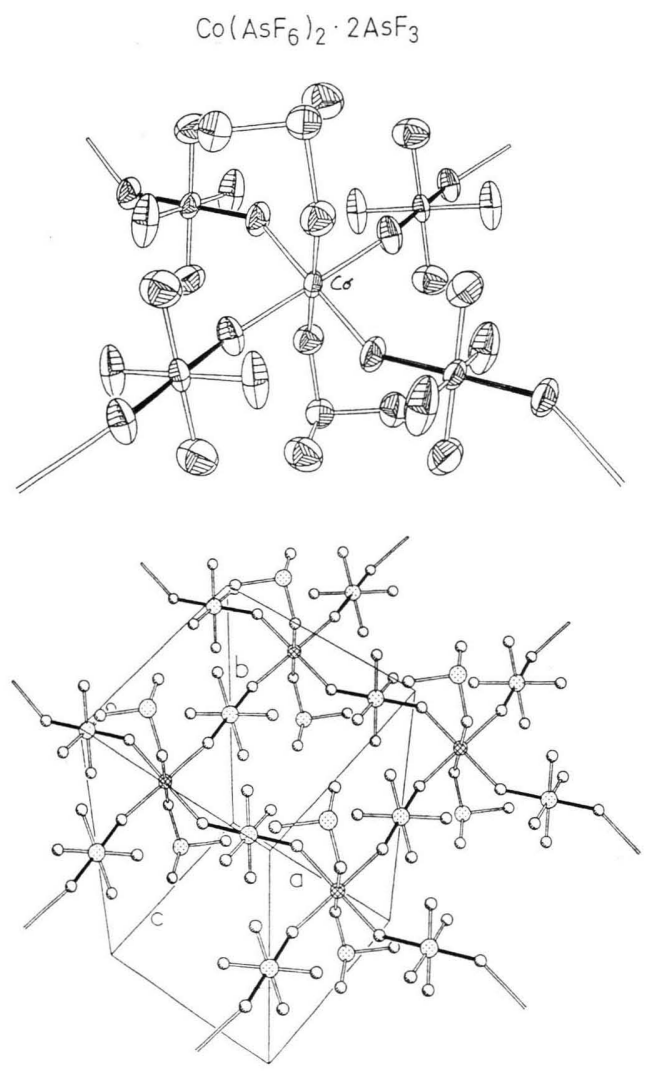

Fig. 1. Closer and more distant view of the crystal structure of $\mathrm{Co}\left(\mathrm{AsF}_{6}\right)_{2} \cdot 2 \mathrm{AsF}_{3}$. 


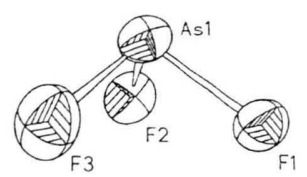

\begin{tabular}{|l|l|}
\hline As1-F1 & $180.2(4) \mathrm{pm}$ \\
\hline As1-F2 & $169.3(6)$ \\
\hline As1-F3 & $169.8(6)$ \\
\hline & \\
\hline F1-As1-F2 & $91.9(2)^{\circ}$ \\
\hline F1-As1-F2 & $92.0(2)$ \\
\hline F1-As1-F2 & $94.5(3)$ \\
\hline & \\
\hline As2-F4 & $176.7(4) \mathrm{pm}$ \\
\hline As2-F5 & $167.9(5)$ \\
\hline As2-F6 & $167.8(6)$ \\
\hline & \\
\hline F4-As2-F5 & $89.7(2)^{\circ}$ \\
\hline F4-As2-F6 & $90.6(3)$ \\
\hline F5-As2-F6 & $90.3(4)$ \\
\hline & \\
\hline As3 - F7 & $177.8(4) \mathrm{pm}$ \\
\hline As3 - F8 & $168.0(5)$ \\
\hline As3 - F9 & $169.0(5)$ \\
\hline & \\
\hline F7-As3-F8 & $90.1(2)^{\circ}$ \\
\hline F7-As3-F9 & $89.1(2)$ \\
\hline F8-As3-F9 & $90.4(3)$ \\
\hline & \\
\hline
\end{tabular}

Fig. 2. Details of the crystal structure with selected dimensions.

groups, located centrosymmetrically above and below the square lattice plane, are additionally coordinated to each $\mathrm{Co}$ atom. They are incorporated into the structure in a way as if they were solvent molecules. Co and As atoms lying on the plane have octahedral environment of $\mathrm{F}$ atoms.

Figure 2 shows the coordination of three chemically inequivalent As atoms of the complex, which are all surrounded only by fluorines. The fluorines in the crystal structure can be divided into two kinds: terminal atoms, coordinated only to As, and bridging atoms, bound to As at one side and to Co at the other. The length of the bond F-As is by about $5 \%$ shorter for the first kind than for the second.

In the $\mathrm{AsF}_{3}$ group the As atoms exhibit valency III, whereas in $\mathrm{AsF}_{6}$ they exhibit valency V. Except for the elongation along the bridging two fluorines the $\mathrm{AsF}_{6}$ octahedra are pretty regular. In the center of a regular equidistant $\mathrm{AsF}_{6}$ octahedron with equal effective charges on $\mathrm{F}$ sites the electric field gradient (EFG) would be zero. A distortion gives rise to a finite EFG.
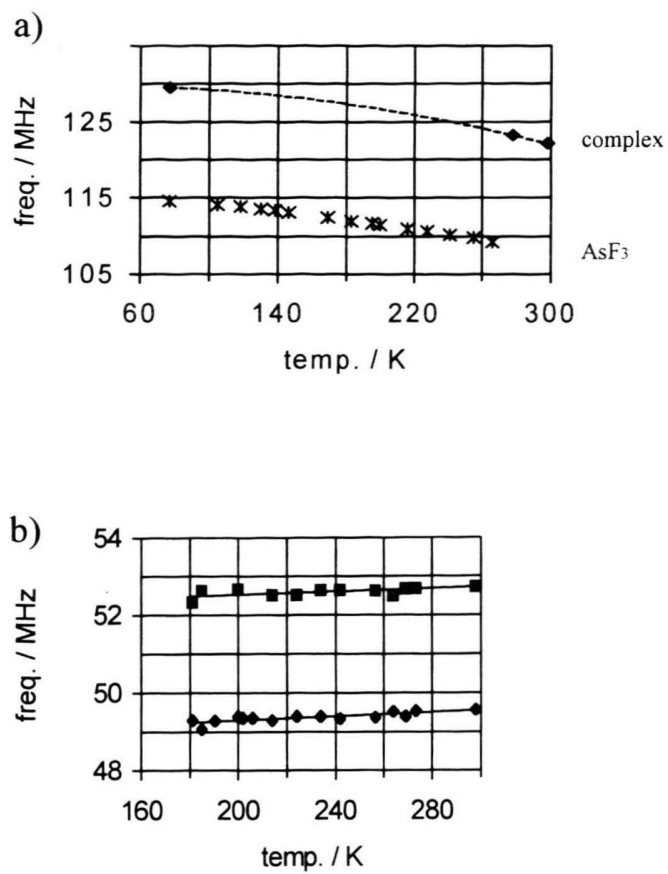

Fig. 3. Temperature dependence of the ${ }^{75} \mathrm{As}$ NQR spectrum: a) high frequency line of the complex compared to the one of pure $\mathrm{AsF}_{3}$; b) low frequency lines of the complex.

\section{Measurements and Discussion}

The powder sample of $\mathrm{Co}\left(\mathrm{AsF}_{6}\right)_{2} \cdot 2 \mathrm{AsF}_{3}$, which is rather aggressive and toxic has been sealed in a quartz tube under argon atmosphere. The measurements of the temperature dependence of ${ }^{75} \mathrm{As} N Q R$ have been done using a superregenerative spectrometer. Two lines found at room temperature near $50 \mathrm{MHz}$ are separated by $3 \mathrm{MHz}$, and the third one is at $\sim 122 \mathrm{MHz}$. The search for the free induction decay or the spin echo of the two ${ }^{75} \mathrm{As}$ lines around $50 \mathrm{MHz}$ - though still well within the range of our pulse spectrometer - was not successful in spite of high averaging up to 2000 times. The temperature dependence of all the three NQR lines is shown in Figure 3.

The assignment of the high frequency line $(\sim 122$ $\mathrm{MHz}$ ) is easier if one is aware that the As NQR in pure $\mathrm{AsF}_{3}$ [3] lies only by $\sim 15 \mathrm{MHz}$ lower, which is also shown in Figure 3. This part of the spectrum therefore belongs to the $\mathrm{AsF}_{3}$ ligand (As1 in Figure 2). With changing temperature the As lines of the pure $\mathrm{AsF}_{3}$ and those of $\mathrm{AsF}_{3}$ in the complex $\mathrm{Co}\left(\mathrm{AsF}_{6}\right)_{2} \cdot 2 \mathrm{AsF}_{3}$ behave very similarly and in accordance with the Bayer model. Their frequency difference remains almost constant down to $77 \mathrm{~K}$. This 
similarity could be interpreted by partly similar vibrational modes which average the EFG tensor. Too much similarity is not expected because one $\mathrm{F}$ atom in the $\mathrm{AsF}_{3}$ ligand is bound also to a Co atom and therefore has a longer distance from As. Unfortunately, the superregenerative spectrometer does not provide reliable line shape measurements, and conclusions about local magnetic fields are not possible so far. It is interesting that the intensity of the high frequency line gradually increases with decreasing temperature.

To derive some conclusions from the comparison of the relative positions of the NQR frequency in the pure $\mathrm{AsF}_{3}$ and the corresponding one in the complex, more EFG components should be known (direction, asymmetry parameter). Roughly it can be said for both cases that the prevailing contribution to the EFG should come from the lone pair orbital. The contributions of the other three bonding $\mathrm{sp}^{3}$ orbitals would cancel to zero if they had equal occupation and their axes were rectangular to each other (cf. the $\mathrm{AsF}_{3}$ group interbond angles, Figure 2).

The remaining part of the As NQR spectrum, belonging to the $\mathrm{AsF}_{6}$ groups with $\mathrm{As}(\mathrm{V})$ (As2 and $\mathrm{As} 3$ in Fig. 2), lies at lower frequencies around $50 \mathrm{MHz}$ and consists of two lines in accordance with the structure. Their temperature coefficients are approximately equal, positive and very small, only $+2 \mathrm{kHz} / \mathrm{K}$. Below $180 \mathrm{~K}$ both lines disappear in the noise (Figure $3 \mathrm{~b}$ ).

The octahedral coordination of As requires six $\mathrm{d}^{2} \mathrm{sp}^{3}$ hybrid bonding orbitals [4]. As already mentioned, the $\mathrm{AsF}_{6}$ octahedra in this structure are nearly regular, the finite EFG value arising mainly from the two elongated bonds, binding the bridging fluorines (As-F-Co bonds, bold lines in Figure 1). The remaining four fluorines are approximately equivalent. Therefore in a static formation we expect an almost axially symmetric EFG tensor along the octahedron long axis. The weak temperature dependence of the corresponding NQR frequencies can be explained by small amplitudes of torsional thermal modes which are effective in averaging down the EFG tensor, or by some mechanism which compensates the normal Bayer contribution to the temperature dependence.

[1] H. Borrmann, K. Lutar, and B. Žemva, Inorg. Chem. 36, 880 (1997).

[2] K. Lutar, H. Borrmann, A. Jesih, and B. Žemva, Acta Chim. Slov. 46, 213 (1999).

[3] P. K. Kadaba, J. Slivnik, R. Blinc, J. Pirnat, and Z. Trontelj, Mol. Physics 29, 1485 (1975).

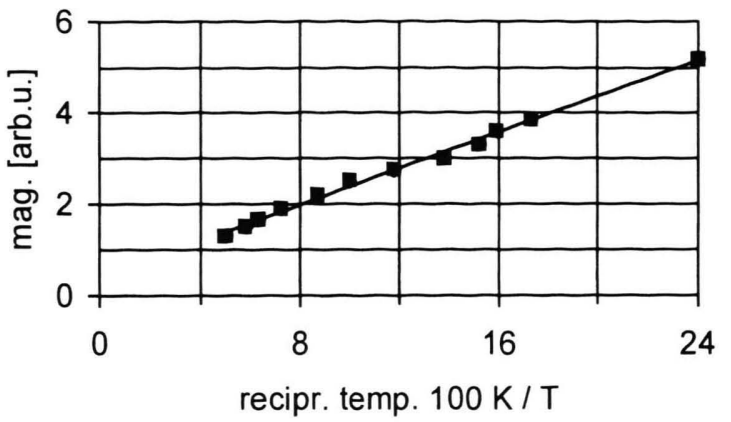

Fig. 4. Temperature dependence of the sample magnetization in a magnetizing field of $13 \mathrm{mT}$ below $20 \mathrm{~K}$.

Such a mechanism could be a gradual elongation of the octahedra with temperature or temperature dependent charge transfer from one side of the bridging fluorine to the other.

An interesting phenomenon is the decreasing intensity of the low frequency lines with decreasing temperature (and simultaneous increasing of the high frequency line intensity). We expect that the future line shape measurements and comparison with the signals in related samples will help us to understand this behaviour.

To estimate the influence of the paramagnetic $\mathrm{Co}$ ions on $\mathrm{As} \mathrm{NQR}$ in $\mathrm{Co}\left(\mathrm{AsF}_{6}\right)_{2} \cdot 2 \mathrm{AsF}_{3}$, the d.c. magnetic susceptibility of the sample has been measured below $25 \mathrm{~K}$, using a home built SQUID magnetometer. The Curie type temperature dependence of the sample magnetization in a magnetizing d.c. field of $13 \mathrm{mT}$ is shown in Figure 4. Comparing this magnetization with that of a standard sample $\left(\mathrm{CuSO}_{4}\right) \cdot 5 \mathrm{H}_{2} \mathrm{O}$ we have estimated the effective magneton number per molecule [5] of the complex as 4.6. This value is very near to the listed experimental value for $\mathrm{Co}^{2+}(4.8)$. It is to be expected that in zero magnetic field at higher temperatures the local magnetic fields at As sites are averaged out by the exchange coupling, so that the NQR signals are observable.

\section{Acknowledgement}

This work is in part supported by the Ministry of Science and Technology of Slovenia.

[4] E. Cartmell and G. W. A. Fowles, Valency and Molecular Structure, Butterworths, London 1966, pp. 181188.

[5] For instance: N. W. Ashcroft and N. D. Mermin, Solid State Physics, Saunders College, Philadelphia 1976, pp. 656-659. 